\title{
Downregulation of SIRT2 by Chronic Stress Reduces Expression of Synaptic Plasticity-related Genes through the Upregulation of Ehmt2
}

\author{
Sung Eun Wang ${ }^{1}$, Seung Yeon $\mathrm{Ko}^{1}$, Sungsin $\mathrm{Jo}^{2}$, Hye-Ryeong Jo ${ }^{1}$, \\ Jinil Han ${ }^{3}$, Yong-Seok Kim ${ }^{1,4}$ and Hyeon Son ${ }^{1,4 *}$ \\ ${ }^{1}$ Hanyang Biomedical Research Institute, Graduate School of Biomedical Science and Engineering, Hanyang University, Seoul \\ 04763, ${ }^{2}$ Hanyang University Hospital for Rheumatic Diseases, Seoul 04763, ${ }^{3}$ Gencurix, Inc, Hanhwan Bizmetro 1, Seoul \\ 08394, ${ }^{4}$ Department of Biochemistry and Molecular Biology, College of Medicine, Hanyang University, Seoul 04763, Korea
}

\begin{abstract}
Silent information regulator 2 (Sirtuin2 / SIRT2) is a $\mathrm{NAD}^{+}$-dependent deacetylase that regulates the cellular oxidative stress response. It modulates transcriptional silencing and protein stability through deacetylation of target proteins including histones. Previous studies have shown that SIRT2 plays a role in mood disorders and hippocampus-dependent cognitive function, but the underlying neurobiological mechanism is poorly understood. Here, we report that chronic stress suppresses SIRT2 expression in the hippocampus. Molecular and biochemical analyses indicate that the stress-induced decrease in the SIRT2 expression downregulates synaptic plasticity-related genes in the hippocampus through the increase of euchromatic histone-lysine N-methyltransferase 2 (Ehmt2) (also known as G9a). shRNA-mediated knockdown of SIRT2 in the dentate gyrus alters the expression of synaptic plasticity-related genes in a way similar to those induced by chronic stress, and produces depression-like behaviors. Our results indicate that SIRT2 plays an important role in the response to stress, thereby modulating depression-like behaviors.
\end{abstract}

Key words: SIRT2, depression, Ehmt2, Synaptic plasticity, Hippocampus

\section{INTRODUCTION}

Silent information regulator proteins (Sirtuins / SIRTs) are nicotinamide adenine dinucleotide $\left(\mathrm{NAD}^{+}\right)$-dependent class III histone deacetylases (HDACs), which are linked to the damage occurring in neurodegenerative diseases [1]. They play key roles in mood disorders [2], and abnormal functioning of SIRT1 me-

Received May 9, 2019, Revised May 31,2019,

Accepted July 8, 2019

* To whom correspondence should be addressed. TEL: 82-2-2220-0626, FAX: 82-2-2220-2418 e-mail:hyeonson@hanyang.ac.kr diates depression-like behavior in the nucleus accumbens and hippocampus of rodents $[3,4]$. However, the function of SIRT2 and its role in depression remain controversial. SIRT2 mRNA is decreased in the peripheral white blood cells of patients with major depressive disorder (MDD) or bipolar disorder (BPD) [5], and SIRT2 overexpression in the hippocampus of mice promotes neurogenesis and reverses chronic unpredictable stress (CUS)-induced depressive-like behaviors [6]. Also SIRT2 expression is increased in the prefrontal cortex of depressed patients and an animal model of chronic social defeat stress [7]. These studies suggest that SIRT2 is involved in the development of depression. However little is known about how SIRT2 regulates stress responses at the molecular level in the hippocampus, which is the region susceptible to 
environmental stress.

Susceptibility or resilience to stress appears to be regulated by epigenetic mechanisms including histone modification [8]. Class I and II HDACs are involved in the behavioral response to chronic stress and antidepressants in rodents, suggesting the possibility of using HDAC inhibitors in patients with treatment-resistant depression [9]. Methylation of histones is an additional epigenetic mechanism involved in depression [10]. Chronic stress induces the expression of euchromatic histone-lysine $\mathrm{N}$-methyltransferase 2 (Ehmt2) involved in complexes repressing transcription [11]. Ehmt2-induced di-methylated histone 3 lysine 9 (H3K9me2) is a marker of repression induced in the hippocampus and amygdala during stress and anxiety [11]. Although Ehmt2 mediates stressinduced depression-like behaviors [11], the associated transcriptional program is poorly understood.

Here we demonstrate that chronic stress-induced downregulation of SIRT2 increases expression of histone methyltransferases in the hippocampus of a mouse depression model, resulting in transcriptional repression of synaptic plasticity-related genes. Furthermore, knockdown of SIRT2 in the dentate gyrus (DG) precipitated depression-like behaviors in mice, accompanied by reduced expression of synaptic plasticity-related genes. These observations reveal a molecular mechanism that explains why hippocampal SIRT2 expression in depressive state impairs synaptic plasticity, and underline the importance of SIRT2 as a potential target for protecting synapses from depression.

\section{MATERIALS AND METHODS}

\section{Mice}

All experiments were conducted with 8-12-week-old male C57BL/6J mice (Charles River Korea, Seoul, Korea). The mice were housed 3-4 per cage in a temperature- and humidity-controlled environment under a 12-h light/dark cycle (lights on 07:00-19:00) with access to food and water ad libitum. All animals were handled in accordance with animal care guidelines of Hanyang University, and all animal experiments were approved by the Institutional Animal Care and Use Committee of Hanyang University (HYIACUC-18-0055).

\section{Culture of primary hippocampal neurons}

Primary hippocampal neuronal were cultured as previously described [12].

\section{Drugs}

For in vitro drug treatment, AGK2 (2-cyano-3-[5-(2,5dichlorophenyl)-2-furanyl]-N-5-quinolinyl-2-propenamide,
A8231, Sigma, MO, USA) was prepared as a $10 \mathrm{mM}$ stock solution in dimethyl sulfoxide (DMSO) and diluted in fresh medium at a final concentration of $5 \mu \mathrm{M}$. Primary hippocampal neurons were treated with AGK2 for $72 \mathrm{~h}$.

\section{Chromatin immunoprecipitation assays (ChIP)}

ChIP assays were performed as previously described [12]. Mouse hippocampal tissue and cultured hippocampal neurons were chemically cross-linked by the addition of formaldehyde solution and then sonicated. The resulting extract was incubated with $5 \mu \mathrm{g}$ of the appropriate antibody and Protein G Agarose beads (Roche, Mannheim, Germany). The antibodies used are anti- $\triangle$ FosB (Cell signaling, MA, USA, \#14695), anti-CoREST (Millipore, CA, USA, $\#$ 07-455) and anti-H3K9me2 (Cell signaling, MA, USA, \#4658). Immunoprecipitated DNA samples were resuspended in distilled $\mathrm{H}_{2} \mathrm{O}$ and used in real-time PCR (CFX96 Touch $^{\mathrm{TM}}$ Real-Time PCR Detection System, Bio-Rad Laboratories, CA, USA), using the following primer pairs corresponding to mouse gene promoter regions: Actb (Actin), 5'-GCCGCTGTGGCGTCCTATAA-3' and 5'- TGAGGTACTAGCCACGAGAG-3'; Sirt2, 5'-GCACAAAAACCAGACCCAAA-3' and 5'-TTGTACCCGGGATTTTCTTG-3'; Ehmt2, 5'-AGATGCCGAATTGGCCTT GG-3' and 5'-TGCCTGTATGGTTGCTCACA-3'; Scmhl, 5'-TGGGTATTAGGGGG AAACTGTA-3' and 5'- TAAGGTTGACAGAATCCTGGGC-3'; Egr1, 5'-CGCCTTA TATGGAGTGGCCC-3' and 5'-AGGCTCCTGGAAAGCCTAGTA-3'; Synaptophysin, 5' - TGCCAACAAGACCGAGAGTG-3' and 5'-TTCGGCTGACGAGGAGTAGT-3'; Synapsin 1, 5'-CCAGCTCAACAAATCCCAGTC-3' and 5'-GCGGATGGTCTCAGCT TTCA-3'. Primer sequences are shown in below. Promoter enrichment was assessed and normalized to the values obtained with the Actin promoter.

\section{Immunohistochemistry and Western blot analysis}

Mice were perfused with 4\% paraformaldehyde in PBS for 20 min and processed for histology. Immunofluorescence labeling and Western blot analysis were performed as previously described [12]. The primary antibodies used for immunohistochemistry and Western blot analysis were as follows: $\beta$-actin (Santa Cruz, sc47778), $\Delta$ FosB (Cell signaling, \#14695), SIRT2 (Millipore, 09-843), Egr 1 (Cell signaling, \#4153), Tuj1 (Covance, MMS-435P), GluR1 (Upstate, \# 06-306), GluR2 (Millipore, AB1768-I), NR1 (Upstate, \#06-311), PSD95 (abcam, ab18258), Synaptophysin (abcam, ab32127), Synapsin 1 (Cell signaling, \#5297), CoREST (Millipore, \#07-455), and GFP (Roche Applied Sciences, 11814460 001). 


\section{Co-immunoprecipitation}

Hippocampal neuron lysates were generated by using lysis buffer (Cell Signaling, MA, USA) contained protease inhibitor and phosphatase inhibitor I and II (Sigma). $2 \mu$ g antibody (CoREST, Millipore, CA, USA, \#07-455) or rabbit IgG (Millipore, CA, USA) was added to lysates containing $500 \mu \mathrm{g}$ protein at $4^{\circ} \mathrm{C}$ overnight [12], which was followed by immunoprecipitation with $30 \mu \mathrm{l}$ Protein $\mathrm{G}$ Agarose beads (Roche, Mannheim, Germany). Beads were washed five times with HNTG buffer (20 mM HEPES pH 7.5, $150 \mathrm{mM}$ $\mathrm{NaCl}, 0.1 \%$ Triton X-100 and 10\% glycerol). The bound proteins were eluted with 2X Laemmli sample buffer (126 mM Tris-HCl, $20 \%$ glycerol, $4 \%$ SDS, $0.02 \%$ bromophenol blue) and analyzed by Western blotting.

\section{RT-PCR and qPCR}

RNA was extracted from hippocampi with Trizol reagent (Sigma), and reverse transcribed with Improm-II (Promega, WI, USA), $1 \mu \mathrm{g}$ of total RNA and oligonucleotide-dT primer. Quantitative real-time PCR (qPCR) was performed on a CFX96 Touch $^{\mathrm{TM}}$ Real-Time PCR Detection System (Bio-Rad Laboratories) using the primer sets of 5'-AGCCAACCATCTGCCACTAC-3' and 5'-CCAGCCCATCGTGTA TTCTT-3' for Sirt2, 5'-AGGCAGAGCTGGAGTCGGAGAT-3' and 5'-GCCGAGGA CTTGAACTTCACTCG-3' for $\triangle F o s B$, 5'-GACGAGTTATCCCAGCCAAA-3' and 5'-GGCAGAGGAAGACGATGAAG-3' for Egr1, 5'CACCAGGATGAAGACAAGCA-3' and 5'-GTCGTTGTTGAGCAGGAG-3' for Synapsin 1, 5'-TGCCAACAAGACGGA G-3' and 5'-GGCGGATGAGCTAACT-3' for Synaptophysin, 5'-TTCTTCAGCTCCA GGGACAT-3' and 5'-AGAATCGGTCACCGTAGTCA-3' for Ehmt2, and 5'-CACCT TGCTCTGACCTCTGAC-3' and 5'-AATTCTAGCTGGATGGGAGTGA-3' for $S c m h 1$. Ct values for each sample were obtained using CFX Manager Software version 3.0 (Bio-Rad Laboratories). Gene expression was normalized to the amount of $\beta$-actin mRNA. Normalized expression values were averaged, and average fold changes were calculated.

\section{Construction of a short hairpin RNA (shRNA)-expressing vector, and lentivirus production}

For Sirt2 gene silencing, XhoI-HpaI fragment including SIRT2 shRNA (shSIRT2; sense, 5'-TGCCTCTATGC AAACCTGGAGAAT TCAAGAGAT TCTCCAGGTTTGCATAG AGGTTTTTTC-3' and antisence, 5'-TCGAGAAAAAACCTCTATGCA AACCTGGA GAATCTCTTGAATTCTCCAGGTTTGCATAGAGGCA-3') were annealed and cloned into pLB lentiviral vector (Addgene, \#11619) [13]. Luciferase shRNA (shLuc) as control vector was previously described
[12]. The shSIRT2 plasmid was confirmed by DNA sequencing (Macrogen, Korea). Human embryonic kidney epithelial 293T cells (ATCC) were maintained in DMEM (Welgene, LM00101) supplemented with 10\% fetal bovine serum (JRS, 43640), 1\% penicillin/streptomycin (Welgene, LS202-02) as growth media. 293 T cells were transfected using the calcium phosphate method [14]. $2 \times 10^{6}$ cells of 293 T were seeded in $100 \mathrm{~mm}$ dish at $60 \sim 70 \%$ confluence the day before transfection. To produce virus, $12 \mu \mathrm{g}$ shSIRT2 or shLuc plasmids were transiently co-transfected with the compatible packaging plasmids, $8 \mu \mathrm{g}$ psPAX2 (Addgene, \#12260) and $6 \mu \mathrm{g}$ pMD2.G (Addgene, \#12259) into 293T cells using $\mathrm{CaPO}_{4}$-DNA precipitate method. After $12 \mathrm{~h}$, the media was changed to DMEM growth medium supplemented with 20\% BSA (Sigma, A7906) and 1M HEPES (Sigma, H3375). After 48 h posttransfection, the media was collected, centrifuged at $1,500 \mathrm{rpm} 4^{\circ} \mathrm{C}$ for $5 \mathrm{~min}$ to remove cell debris, and sterilized using $0.45 \mu \mathrm{m}$ low protein binding syringe filters (ADVANTEC, 25CS045AS). For virus concentration, collected lentiviral supernatant was added with 5X PEG solution (Sigma, P5413) and incubated for overnight at $4{ }^{\circ} \mathrm{C}$. Next day, the mixture was centrifuged at 3,000 rpm $4{ }^{\circ} \mathrm{C}$ for 30 min and the pellet was incubated with $1 \mathrm{X}$ PBS containing $1 \%$ BSA on the shaker at $4^{\circ} \mathrm{C}$. For virus transduction, concentrated virus involving $8 \mu \mathrm{g} / \mathrm{ml}$ protamine sulfate (Sigma, P4020) was transferred to in vivo or in vitro, and performed further experimental analysis.

\section{Virus-mediated gene transfer}

Mice were anesthetized with a mixture of ketamine (100 mg/ $\mathrm{kg}$ ) and xylazine $(10 \mathrm{mg} / \mathrm{kg}$ ) and prepared for stereotaxic surgery. Thirty-three gauge syringe needles (Hamilton, NV, USA) were used to bilaterally infuse $3 \mu$ of virus into the dorsal hippocampus at a rate of $0.1 \mu \mathrm{l} / \mathrm{min}$ at the stereotaxic coordinates -2.0 anteroposterior (AP), \pm 1.5 mediolateral (ML), -2.4 dorsoventral (DV) (mm from bregma, horizontal skull).

\section{Chronic stress procedures}

Chronic unpredictable stress (CUS) experiments were performed as described previously. Mice were exposed to a sequence of mild and unpredictable stressors for $14 \mathrm{~d}$ [12]. The 11 stressors used to generate the animal model of depression were as previously described [15]. For the chronic restraint stress (CRS) procedure, the mice were restrained horizontally in $50 \mathrm{ml}$ conical centrifuge tubes drilled with holes to allow ample ventilation; the tubes were small enough to restrain the mice so that they were able to breathe but unable to move freely. The mice were stressed daily for $2 \mathrm{~h}$ for $14 \mathrm{~d}$, and provided with food and water ad libitum [16]. 


\section{Assessment of behavior}

On the test day, mice were placed to the testing room and acclimated to the room conditions for at least $1 \mathrm{~h}$. Mice of the same cage were randomly assigned to different experimental groups for behavioral studies, and the order of testing was distributed across groups. All tests were conducted during the dark cycle. After each individual test session, the apparatus was thoroughly cleaned with $70 \%$ alcohol to eliminate any odor and trace of the previously tested mouse. Wherever possible, the experimenter conducting the data analysis was blind to the treatment conditions of the animals.

\section{Locomotor activity test (LMA)}

Total locomotor activity was measured for 5 min with an ANYmaze video tracking system (Stoelting Co., IL, USA) in a plastic box $(50 \times 50 \times 20 \mathrm{~cm})$ and conducted as previously described [12].

\section{Forced swim test (FST)}

FST was conducted as previously described. Mice were placed in a cylinder (height: $30 \mathrm{~cm}$, diameter: $15 \mathrm{~cm}$ ) filled with water at $24 \pm 1^{\circ} \mathrm{C}$ and a depth of $12 \mathrm{~cm}$ for $6 \mathrm{~min}$. The immobility time in the last $4 \mathrm{~min}$ of the $6 \mathrm{~min}$ test period were measured [12].

\section{Learned helplessness test (LHT)}

The learned helplessness procedure was based on previously described procedures. Mice were placed on a commercial shuttle box divided into two equal compartments by a central barrier (Gemini Avoidance System, San Diego Instruments) and then given inescapable footshocks ( $0.3 \mathrm{~mA}, 180$ scrambled footshocks). The mean escape latency over 30 successive escape trials $(0.3 \mathrm{~mA}$, 25 -sec maximum duration) was measured $[12,17]$.

\section{Microarray dataset analysis}

The following analyzing method was previously described [18]. The gene expression microarray dataset was obtained from the Gene Expression Omnibus (GEO, http://www.ncbi.nlm.nih.gov/ geo/) with accession number GSE53987. It is described in detail in Lanz et al. (2015) [19]. Briefly, frozen sections of human brain were dissected to obtain tissue samples of hippocampus, Brodmann Area 46 and associative striatum. Total RNAs were isolated from the samples and microarray experiments were conducted with an Affymetrix platform (U133Plus-v2 Affymetrix whole genome microarray chips). The gene expression data were processed with Robust Multi-array Average (RMA). To identify genes differentially expressed in the hippocampi of controls and subjects with MDD, this study focused on a subset of the samples collected from the hippocampi; thus the samples were filtered to exclude samples of low quality (Brain $\mathrm{pH} \leq 6.5$, RNA Integrity Number $(\mathrm{RIN}) \leq 6.0$,
Postmortem interval (PMI) $<24$ hours). Next, the expression data were normalized by quantile normalization, and $\log 2$ transformed. Differentially expressed genes were identified using the LIMMA (Linear modeling of Microarray data) package [20]. Because of the small sample size, the usual statistical criteria used in microarray analysis (i.e., false discovery rate, $\mathrm{FDR}<0.05$ ) was not inappropriate for revealing differences between groups. Instead, p-values were used to obtain potentially interesting genes ( $\mathrm{p}$-value $<0.05$ ). All data analyses and visualizations were conducted using $\mathrm{R} 3.4 .3$ (www.r-project.org).

\section{Statistical analyses}

Data distributions were checked for normality and equal variance. All experiments were carried out at least three times, and data consistency was observed in repeated experiments. GraphPad Prism5.0 (GraphPad Software, Inc., La Jolla, CA, USA) was used for statistical analysis. $\mathrm{p}<0.05$ was considered statistically significant. Biochemical data are presented as means \pm s.e.m. of multiple independent experiments performed in triplicate.

\section{RESULTS}

\section{Chronic stress downregulates exprssions of $\triangle F o s B$ and SIRT2 in the hippocampus of mice}

SIRT2 expression is decreased in the blood of depressive-state MDD patients, and restored in the remissive state [5]. To examine the role of SIRT2 in the hippocampus following exposure to stress, we obtained mice that had been exposed to CUS or CRS for 2 weeks. We confirmed the reduced levels of SIRT2 in the hippocampus of the CUS (Fig. 1A) and CRS (Fig. 1B) models. Expression of delta FosB $(\Delta \mathrm{FosB})$, a transcription factor that is involved in stress [21] and known to directly regulate SIRT2 expression [22], was reduced in both depression models (Fig. 1A and 1B). To investigate direct regulation by $\Delta \mathrm{FosB}$ of the Sirt 2 promoter in the hippocampus, we performed ChIP assays on the hippocampi of mice subjected to stress. We found that enrichment of $\Delta$ FosB on the Sirt2 promoter was significantly decreased in mice under CUS or CRS (Fig. 1C and 1E), with a concomitant reduction of Sirt2 mRNA in hippocampi (Fig. 1D and 1F). Together, these results suggest that chronic stress downregulates SIRT2 expression possibly due to reduced $\Delta$ FosB in the hippocampus.

\section{Expression of SIRT2 is decreased in the hippocampus in $M D D$}

To see if our results could be translated to human, we evaluated SIRT2 mRNA levels in post-mortem hippocampal tissue in MDD patients. The gene expression dataset from GSE53987 was used 
A

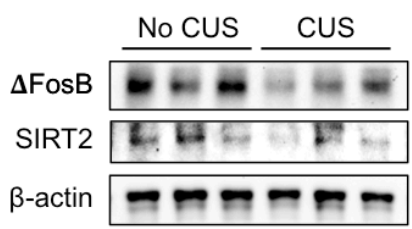

C

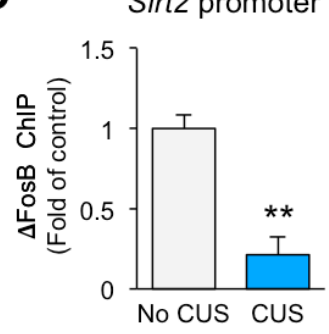

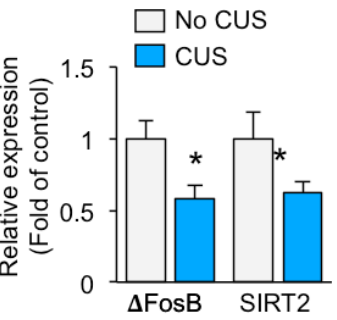

D

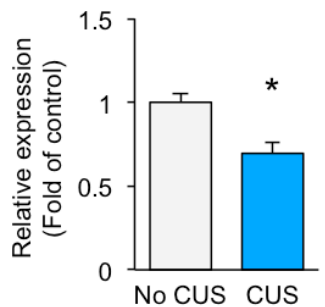

B

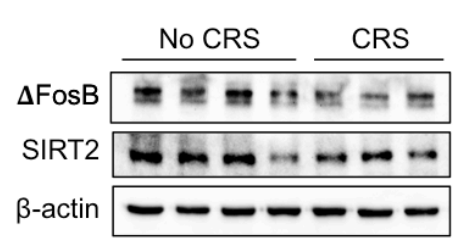

E

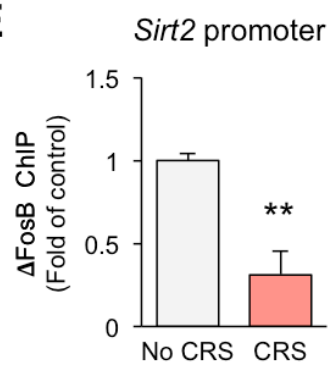

H

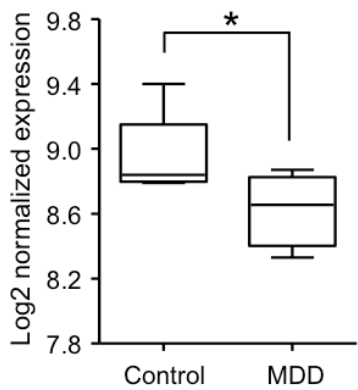$$
\text { Control MDD }
$$

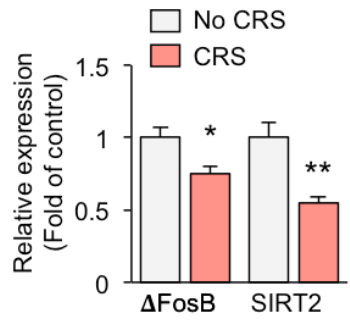

F

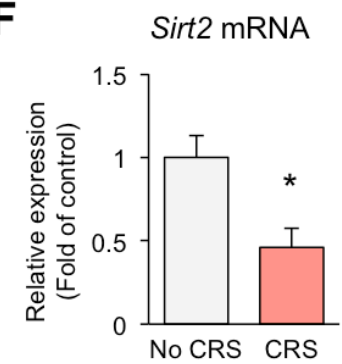

G

\begin{tabular}{cccccc}
\hline \multirow{2}{*}{ Symbol } & \multicolumn{3}{c}{ Expression (log 2 scale) } & \multirow{2}{*}{ P value } \\
\cline { 2 - 5 } & Average & Control & MDD & Difference & \\
\hline SIRT1 & 7.39 & 7.42 & 7.32 & 0.10 & 0.419 \\
SIRT2 & 8.87 & 8.97 & 8.63 & 0.34 & 0.013 \\
SIRT3 & 8.21 & 8.20 & 8.24 & -0.04 & 0.729 \\
SIRT4 & 4.32 & 4.37 & 4.20 & 0.17 & 0.171 \\
SIRT5 & 6.54 & 6.53 & 6.57 & -0.04 & 0.694 \\
SIRT6 & 6.81 & 6.81 & 6.79 & 0.02 & 0.829 \\
SIRT7 & 6.71 & 6.71 & 6.71 & 0.00 & 0.966 \\
\hline
\end{tabular}

Fig. 1. SIRT2 expression is decreased in the hippocampus of an animal model of depression and MDD patients. (A) Representative blots in hippocampus extracts (left). Quantification of the experiments showed decreased levels of $\Delta$ FosB and SIRT2 in the hippocampus of mice under CUS ( $n=3$ per group) (right). (B) Representative blots of hippocampus extracts (left). Quantification showed decreased levels of $\Delta$ FosB and SIRT2 in the hippocampus of mice under CRS ( $n=3 \sim 4$ per group). (C) ChIP assays. Binding of $\Delta$ FosB to the Sirt2 promoter was decreased in the hippocampus of mice under CUS ( $n=3$ per group). (D) Sirt 2 and $\beta$-actin mRNA levels measured by qPCR in hippocampi of mice under CUS ( $n=3$ per group). (E) ChIP assays. Binding of $\Delta$ FosB to the Sirt 2 promoter was decreased in the hippocampus of mice under CRS ( $n=3$ per group). (F) Sirt 2 and $\beta$-actin mRNA levels were quantified by qPCR in hippocampus of mice under CRS ( $n=3$ per group). (G) Microarray analysis (dataset accession number GSE53987) of post-mortem MDD hippocampal samples confirmed the reduction in SIRTs. (H) SIRT2 was down-regulated in post-mortem hippocampal tissue from subjects with MDD. Using appropriate sample selection criteria, we performed gene expression analyses on 14 hippocampus samples; 4 from patients with MDD and 10 controls. Data are mean \pm s.e.m. Unpaired $t$ tests; ${ }^{*} \mathrm{p}<0.05,{ }^{* *} \mathrm{p}<0.01$.

to identify differences in gene expression in the hippocampus between patients with MDD and controls. Using the relevant sample selection criteria we performed gene expression analyses on a cohort of 14 hippocampus samples: 4 from patients with MDD and 10 from controls. As shown in Fig. 1G, there were no significant differences in demographic characteristics between the subjects with MDD and the controls. SIRT2 mRNA was significantly lower in the hippocampi of the MDD patients than in those of the controls (Fig. 1H). No significant differences were observed for other SIRTs (Fig. 1G). These results indicate that stress-induced downregulation of SIRT2 may be associated with the development of depression.

\section{Downregulation of SIRT2 reduces the expression of synap- tic plasticity-related genes in primary hippocampal neu- rons}

To investigate whether knockdown of SIRT2 affects expression of synaptic plasticity-related genes, we introduced lenti-shSIRT2 into hippocampal primary neurons to reduce SIRT2 expression. SIRT2 expression was markedly attenuated at both mRNA and protein levels (Fig. 2A and 2D). $\Delta$ FosB is known to directly regulate SIRT2 expression but we also found that SIRT2 knockdown decreased expression of $\Delta$ FosB (Fig. $2 B$ and 2D). Early growth response protein 1 (Egrl) is thought to play a key role in inducing synaptic plasticity-related genes [23], and SIRT2 knockdown 

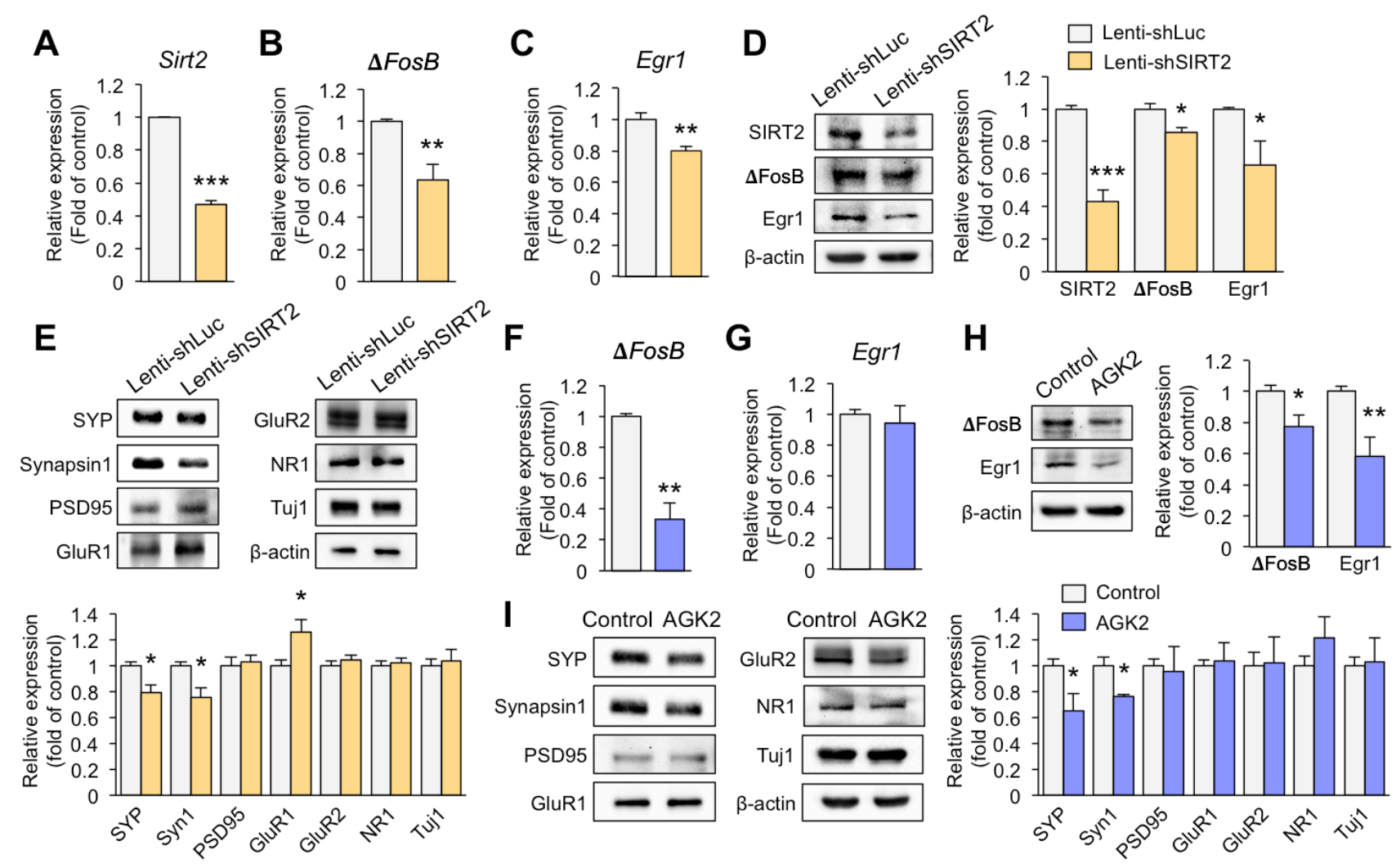

Fig. 2. Downregulation of SIRT2 decreases expression of synaptic plasticity-related genes in hippocampal neurons. (A) To assess the efficiency of lentishSIRT2, Sirt2 and $\beta$-actin mRNA levels were quantified by qPCR $(n=3)$. (B, C) Levels of $\triangle F o s B$ and Egr 1 mRNAs in mouse hippocampal primary neurons were assayed by qPCR $(n=3)$. (D) Western blot analysis showed that expression levels of SIRT2, $\Delta$ FosB, and Egr 1 were decreased by lenti-shSIRT2 infection in hippocampal primary neurons $(n=3)$. (E) lenti-shSIRT2 infection reduced protein levels of Synaptophysin (SYP) and Synapsin1 (Syn1) ( $n=3)$. (F I) The SIRT2-specific inhibitor, AGK2, $(5 \mu \mathrm{m}, 72 \mathrm{~h})$ mimicked the effect of lenti-shSIRT2 infection in primary hippocampal neurons. (F, G) Levels of $\triangle F o s B$ and Egr 1 mRNAs in mouse hippocampal primary neurons were assayed by qPCR $(n=3)$. (H, I) Western blot analysis showed that expression levels of $\Delta$ FosB, Egrl, and pre-synaptic molecules were decreased by AGK2 treatment in hippocampal primary neurons ( $n=3 \sim 4)$. Data are mean \pm s.e.m. Unpaired $t$ tests; ${ }^{*} \mathrm{p}<0.05,{ }^{* *} \mathrm{p}<0.01$.

reduced the expression of Egrl together with the pre-synaptic molecules, Synaptophysin and Synapsin 1 (Fig. 2C E). In addition, expression of glutamate receptor 1 (GluR1) was increased in lenti-shSIRT2 infected neurons compared to lenti-shLuc infected neurons (Fig. 2E), consistent with an elevated level of GluR1 as a result of its increased stability due to reduced deacetylation by SIRT2 [24]. To see if a SIRT2 inhibition had similar effects to SIRT2 knockdown, we treated primary hippocampal neurons with AGK2 ( $5 \mu \mathrm{M}, 72 \mathrm{~h})$, an inhibitor of SIRT2, and found that expression levels of $\triangle \mathrm{FosB}$ and pre-synaptic molecules including Synaptophysin and Synapsin 1 were decreased, while that of Egrl did not (Fig. 2F I). Together, these results indicate that SIRT2 regulates the expression of transcription factors and pre-synaptic molecules that play important roles in resilience to stress.

\section{SIRT2-mediated suppression of Ehmt2 increases presynap- tic gene expression in hippocampal neurons}

It is well known that SIRT2 targets lysines in various cytosolic proteins [25]. However, we have focused on the function of SIRT2 in the nucleus, where transcriptional regulation occurs. SIRT2 is recruited to specific loci by co-repressor element-1 silencing transcription factor (CoREST) [26]. To study whether the inhibition of synaptic plasticity-related genes is regulated by SIRT2 knockdown, we first examined the association of SIRT2 with its corepressor and observed decreased association in primary hippocampal neurons infected with lenti-shSIRT2 compared to neurons infected with lenti-shLuc (Fig. 3A). Given that SIRT2-CoREST corepressor complexes would inhibit the transcription of genes [26], we would expect that expression of SIRT2 target genes was increased in lentishSIRT2 infected neurons. We found that coimmunoprecipitation 


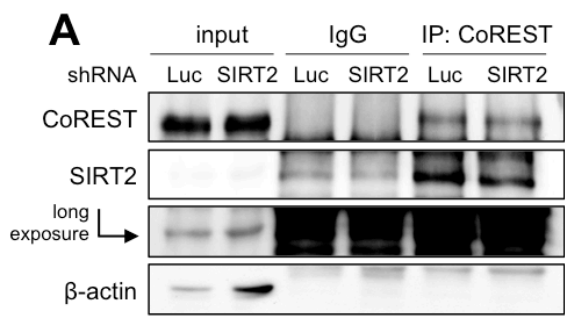

E

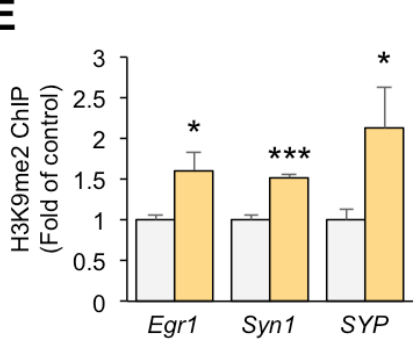

$\mathrm{H}$

H Ehmt2 (G9a)

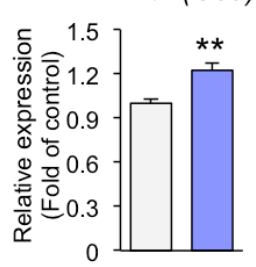

$\mathbf{F}$

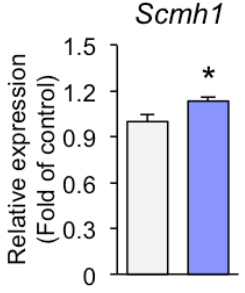

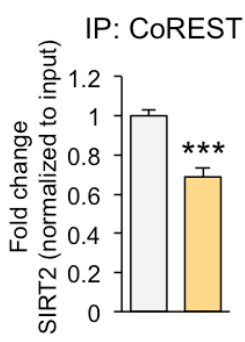

Synapsin 1
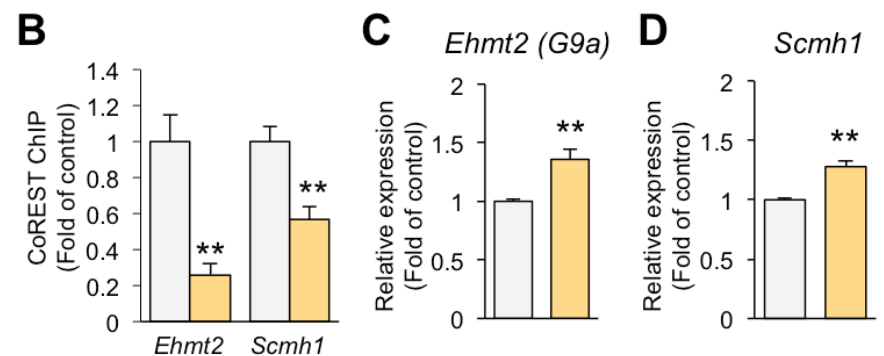

\section{G}
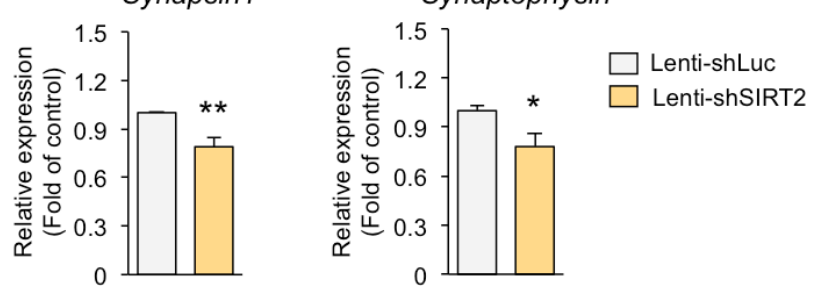

Synaptophysin

$\mathrm{J}$
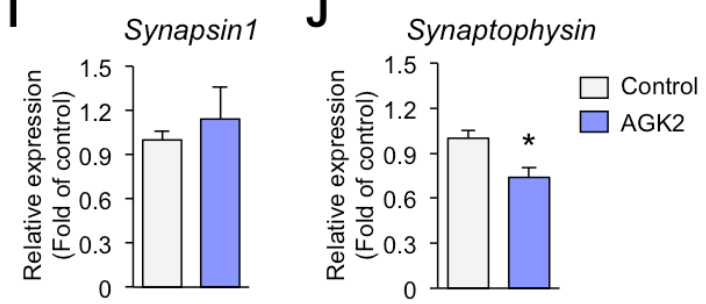

Fig. 3. Downregulation of SIRT2 increases the levels of Ehmt2 and H3K9me2 at the promoters of presynaptic genes in hippocampal neurons. (A) Representative blots of SIRT2 and CoREST following immunoprecipitation of primary hippocampal neuron extracts (left). Protein levels were normalized to the abundance in SIRT2 input (right). Reduced SIRT2 binding to CoREST in lenti-shSIRT2 infected neurons ( $n=4)$. (B) ChIP assays. SIRT2 knockdown decreased the association of SIRT2-CoREST complexes with the Ehmt2 and $S \mathrm{cmhl}$ promoters in hippocampal primary neurons ( $n=3$ ). (C, D) Levels of Ehmt2 and $S c m h 1$ mRNAs in mouse hippocampal primary neurons were assayed by qPCR ( $n=3)$. (E) ChIP assays. SIRT2 knockdown increased H3K9me2 levels of Egr1,Synapsin1 (Syn1) and Synaptophysin (SYP) promoters in hippocampal primary neurons (n=3). (F, G) Synapsin1 and Synaptophysin mRNAs in the mouse hippocampal primary neurons were assayed by qPCR $(n=3)$. (H) Effect of AGK2 treatment on levels of Ehmt2 and $S c m h 1$ mRNAs in mouse hippocampal primary neurons was assayed by qPCR ( $n=3)$. (I, J) Effect of AGK2 treatment on levels of Synapsin1 and Synaptophysin mRNAs in mouse hippocampal primary neurons was assayed by qPCR $(n=3)$. Data are mean \pm s.e.m. Unpaired $t$ tests; ${ }^{*} \mathrm{p}<0.05$, ${ }^{* *} \mathrm{p}<0.01$, ${ }^{* * *} \mathrm{p}<0.001$

of SIRT2 and CoREST was reduced in neurons that were infected by lenti-shSIRT2 compared to neurons infected with lenti-shLuc (Fig. 3A). CoREST binding to the promoter region of Ehmt2 and Scm Polycomb Group Protein Homolog 1 (Scmh1) was decreased in neurons infected by lenti-shSIRT2 (Fig. 3B). Consistently, mRNA levels of Ehmt2 and Scmh1, two histone methyltransferases that targeted by SIRT2, were increased in primary hippocampal neurons infected with lenti-shSIRT2 compared to neurons infected with lenti-shLuc (Fig. 3C and 3D), suggesting that SIRT2 negatively regulates the expression of Ehmt 2 and $\mathrm{Scmh} 1$ although it is not clear whether this is a direct or indirect effect. Furthermore, $\mathrm{H} 3 \mathrm{~K} 9 \mathrm{me} 2$, a known target of Ehmt 2 and a marker of repressed gene repression, was enriched on the promoter regions of Egr1, Synapsin 1, and Synaptophysin (Fig. 3E), consistent with the reduced mRNA levels of Egr1, Synapsin1, and Synaptophysin
(Fig. 2C, 3F and 3G). Likewise, AGK2 induced the mRNA levels of Ehmt 2 and Scmhl along with a decrease of Synaptophysin mRNA (Fig. 3H and 3J). Level of Synapsin I mRNA showed only a tendency to increase in response to AGK2 (Fig. 3I). Collectively, these results suggest that SIRT2 attenuation lowers expression of synaptic plasticity-related genes, and this may, at least in part, contribute to depression-like behaviors.

\section{Knockdown of SIRT2 in the DG induces depression-like be- haviors in mice}

To demonstrate behavioral effects of SIRT2 knockdown, we infused lenti-shSIRT2 bilaterally into the hippocampal DG, which region is vulnerable to stress [27] (Fig. 4A and 4B). SIRT2 expression was reduced at both the mRNA and protein level (Fig. 4C and 4D). We then evaluated whether SIRT2 knockdown increased 


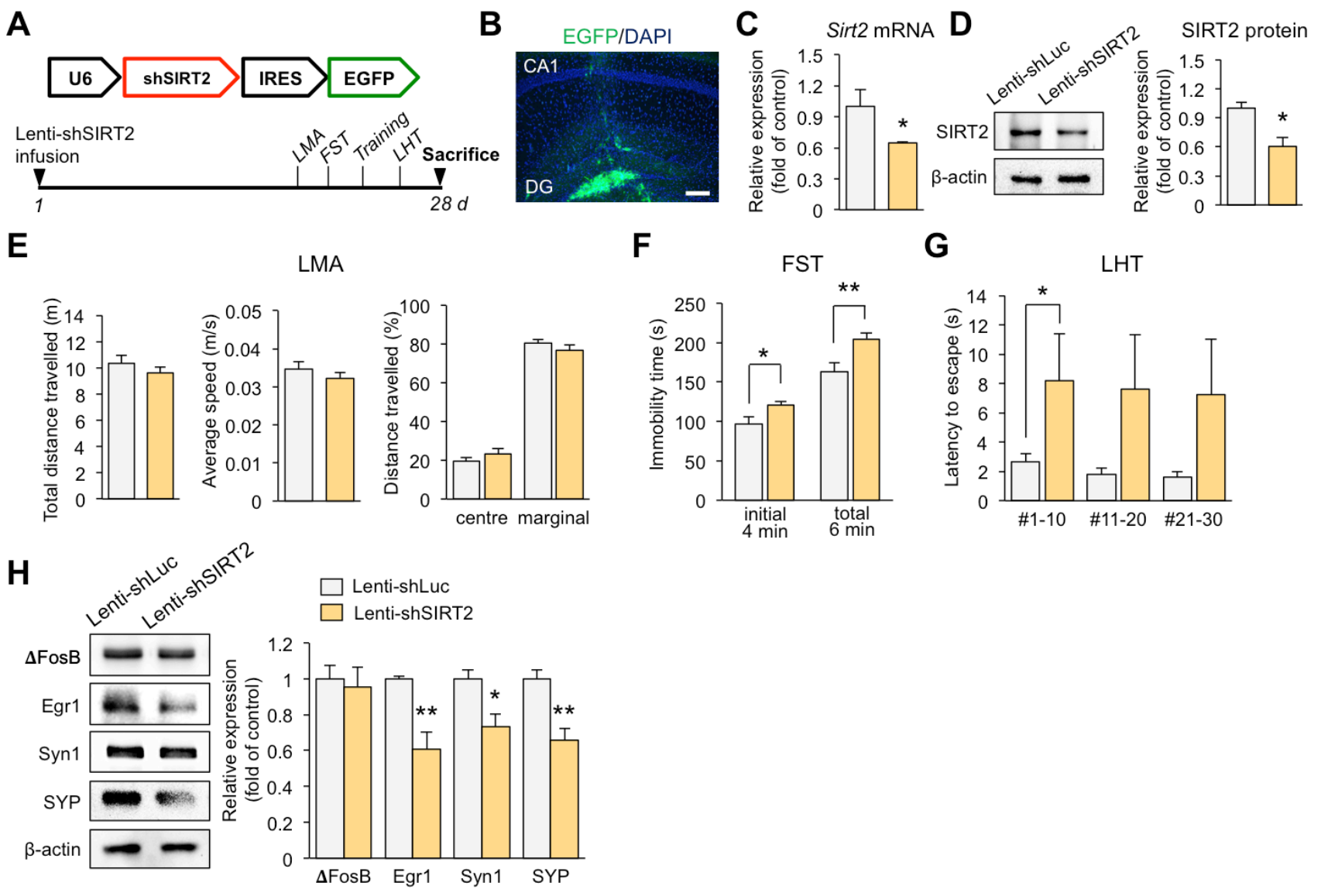

Fig. 4. SIRT2 knockdown is accompanied by depression-like behaviors in mice. (A) The mouse U6 promoter was used to drive the expression of SIRT2shRNA (top). Experimental design (bottom). (B) GFP staining confirmed localization of lentivirus in the DG. (Scale bar, $100 \mu \mathrm{m})$. (C) Mouse Sirt2 and $\beta$-actin mRNA levels were quantified by qPCR ( $n=3$ per group). (D) SIRT2 protein was quantified by Western blotting ( $n=3$ per group). (E) LMA. Total distance, average speed, and distances travelled in the center or margin zone were scored at 5 min intervals $(n=11,10)$. (F) FST. Animals infused with lenti-shSIRT2 displayed depression-like behaviors compared with animals infused with lenti-shLuc $(n=11,11)$. (G) LHT. lenti-shSIRT2-infused mice had a longer latency to escape than animals infused with lenti-shLuc in trials 1-10 $(n=10,8)$. (H) Representative blots of DG lysates (left). Quantification of the results (right) $(n=3)$. Data are means \pm s.e.m. Unpaired $t$ tests; ${ }^{*} \mathrm{p}<0.05,{ }^{* *} \mathrm{p}<0.01$.

stress-induced behaviors, as indicated by reduced responsiveness in the FST and the LHT, two widely used models of behavioral despair. We found that immobility time and the latency to escape increased in the lenti-shSIRT2-infused mice (Fig. 4F and 4G). There was no effect on spontaneous locomotor activity, including average speed, total distance travelled, and distance travelled from the center or margin, indicating that the effects in the FST were not due simply to inhibition of mobility (Fig. 4E). Consistent with the in vitro experiments, levels of Egrl and pre-synaptic molecules were reduced in the lenti-shSIRT2-infused DG. SIRT2 knockdown resulted in downregulation of $\triangle \mathrm{FosB}$ in vitro, while $\triangle \mathrm{FosB}$ was not affected in DG that infused with lenti-shSIRT2 (Fig. 4H). The difference might be due to the age of neurons (embryonic versus adult), the environment (isolated versus intact) etc. Taken together, these results suggest that SIRT2 knockdown induces depression-like behaviors by downregulating synaptic plasticityrelated genes.

\section{DISCUSSION}

This study demonstrates that CUS downregulates SIRT2, resulting in an increase in the Ehmt2 which functions in the CoREST repressor complex. Ehmt2 targets the promoters of synaptic plasticity-related genes such as Egr1, Synaptophysin and Synap$\sin 1$. These promoters are also marked by $\mathrm{H} 3 \mathrm{~K} 9 \mathrm{me} 2$, leading to a reduction in transcript levels of genes. Collectively, SIRT2 knockdown in the DG results in depression-like behaviors in mice, accompanied by decreased expression of synaptic plasticity-related genes. These findings provide a molecular link between SIRT2 downregulation and impaired synaptic plasticity in the hippocam- 
pus of MDD patients. Therefore, SIRT2 expression may be of critical importance for maintaining synaptic plasticity and promoting resilience to stress.

Here, we show that $\mathrm{H} 3 \mathrm{~K} 9 \mathrm{me} 2$, a marker of repressive genes, accumulates in the promoter regions of Egr1, Synaptophysin, and Synapsin1, which might be regulated via Ehmt2. In addition, Egr1 is a transcription factor that binds to the promoter regions of Synapsin1 and Synpatophysin, which have Egr1-binding consensus sequences [28]. Therefore, downregulation of Egr1 might contribute to the reduced transcript levels of Synapsin 1 and Synaptophysin. Taken together, these findings are in line with the previous suggestion that Egrl positively regulates synaptic plasticity and neuropsychiatric disorders including stress-related mood disorders [23]. With regard to SIRT2 regulation of Ehmt2, we suggest the possibility that SIRT2 might be a key component to maintain CoREST-SIRT2 repressor complex for negatively regulating HMT promoters. Previous studies reported that stability of other CoREST repressor complex could be decreased by degradation/reduction of key component [29]. It would be worthwhile investigating whether downregulation by Ehmt 2 would be sufficient to rescue the SIRT2 knockdown. In fact, Ehmt2 downregulation by Ehmt 2 specific inhibitor reduced anxiety-like behaviors in mice [30]. We are currently investigating whether SIRT2 directly regulates Ehmt 2 and the effects of Ehmt 2 knockdown on animal model of depression.

According to previous reports, SIRT2 has a major role in modulating diverse biological mechanisms in the cytosol, including oxidative stress and inflammatory response in microglia cells associated with CNS disorders [2]. SIRT2 downregulation leads to cognitive impairment through aberrant post-translational modification of the AMPA receptor (AMPAR) in the CNS, which is directly related to depressive states $[24,31]$. The function of SIRT2 in the nucleus is poorly understood; it appears to be a kind of histone deacetylase but its underlying role in depression is unclear. To the best of our knowledge, our findings constitute the first report that downregulation of SIRT2 produces depression-like behaviors via increased expression of histone methyltransferase that represses the promoter regions of synaptic plasticity-related genes in the hippocampus, and therefore, SIRT2 may be an attractive therapeutic target in major depressive disorder.

\section{ACKNOWLEDGEMENTS}

This work was supported by a National Research Foundation of Korea (NRF) grant (No. 2019R1A2C2003616 to H.S.), a Medical Research Center Grant (2017R1A5A2015395 to H.S.) and a Basic Science Research Program Grant (No. 2017R1A6A3A01005765 to
S.E.W.) funded by the Ministry of Science and Technology (MEST), Republic of Korea.

\section{REFERENCES}

1. Min SW, Sohn PD, Cho SH, Swanson RA, Gan L (2013) Sirtuins in neurodegenerative diseases: an update on potential mechanisms. Front Aging Neurosci 5:53.

2. Song J, Kim J (2016) Role of sirtuins in linking metabolic syndrome with depression. Front Cell Neurosci 10:86.

3. Kim HD, Hesterman J, Call T, Magazu S, Keeley E, Armenta K, Kronman H, Neve RL, Nestler EJ, Ferguson D (2016) SIRT1 mediates depression-like behaviors in the nucleus accumbens. J Neurosci 36:8441-8452.

4. Abe-Higuchi N, Uchida S, Yamagata H, Higuchi F, Hobara T, Hara K, Kobayashi A, Watanabe Y (2016) Hippocampal sirtuin 1 signaling mediates depression-like behavior. Biol Psychiatry 80:815-826.

5. Abe N, Uchida S, Otsuki K, Hobara T, Yamagata H, Higuchi F, Shibata T, Watanabe Y (2011) Altered sirtuin deacetylase gene expression in patients with a mood disorder. J Psychiatr Res 45:1106-1112.

6. Liu R, Dang W, Du Y, Zhou Q, Jiao K, Liu Z (2015) SIRT2 is involved in the modulation of depressive behaviors. Sci Rep 5:8415.

7. Erburu M, Muñoz-Cobo I, Domínguez-Andrés J, Beltran E, Suzuki T, Mai A, Valente S, Puerta E, Tordera RM (2015) Chronic stress and antidepressant induced changes in Hdac5 and Sirt2 affect synaptic plasticity. Eur Neuropsychopharmacol 25:2036-2048.

8. Tsankova N, Renthal W, Kumar A, Nestler EJ (2007) Epigenetic regulation in psychiatric disorders. Nat Rev Neurosci 8:355-367.

9. Covington HE 3rd, Maze I, LaPlant QC, Vialou VF, Ohnishi YN, Berton O, Fass DM, Renthal W, Rush AJ 3rd, Wu EY, Ghose S, Krishnan V, Russo SJ, Tamminga C, Haggarty SJ, Nestler EJ (2009) Antidepressant actions of histone deacetylase inhibitors. J Neurosci 29:11451-11460.

10. Sun H, Kennedy PJ, Nestler EJ (2013) Epigenetics of the depressed brain: role of histone acetylation and methylation. Neuropsychopharmacology 38:124-137.

11. Covington HE 3rd, Maze I, Sun H, Bomze HM, DeMaio KD, Wu EY, Dietz DM, Lobo MK, Ghose S, Mouzon E, Neve RL, Tamminga CA, Nestler EJ (2011) A role for repressive histone methylation in cocaine-induced vulnerability to stress. Neuron 71:656-670.

12. Wang SE, Ko SY, Jo S, Choi M, Lee SH, Jo HR, Seo JY, Lee SH, 
Kim YS, Jung SJ, Son H (2017) TRPV1 regulates stress responses through HDAC2. Cell Reports 19:401-412.

13. Narayan N, Lee IH, Borenstein R, Sun J, Wong R, Tong G, Fergusson MM, Liu J, Rovira II, Cheng HL, Wang G, Gucek M, Lombard D, Alt FW, Sack MN, Murphy E, Cao L, Finkel T (2012) The NAD-dependent deacetylase SIRT2 is required for programmed necrosis. Nature 492:199-204.

14. Kwon M, Firestein BL (2013) DNA transfection: calcium phosphate method. Methods Mol Biol 1018:107-110.

15. Schmidt HD, Duman RS (2010) Peripheral BDNF produces antidepressant-like effects in cellular and behavioral models. Neuropsychopharmacology 35:2378-2391.

16. Kim KS, Han PL (2006) Optimization of chronic stress paradigms using anxiety- and depression-like behavioral parameters. J Neurosci Res 83:497-507.

17. Duman CH, Schlesinger L, Kodama M, Russell DS, Duman RS (2007) A role for MAP kinase signaling in behavioral models of depression and antidepressant treatment. Biol Psychiatry 61:661-670.

18. Ko SY, Wang SE, Lee HK, Jo S, Han J, Lee SH, Choi M, Jo HR, Seo JY, Jung SJ, Son H (2019) Transient receptor potential melastatin 2 governs stress-induced depressive-like behaviors. Proc Natl Acad Sci U S A 116:1770-1775.

19. Lanz TA, Joshi JJ, Reinhart V, Johnson K, Grantham LE 2nd, Volfson D (2015) STEP levels are unchanged in pre-frontal cortex and associative striatum in post-mortem human brain samples from subjects with schizophrenia, bipolar disorder and major depressive disorder. PLoS One 10:e0121744.

20. Ritchie ME, Phipson B, Wu D, Hu Y, Law CW, Shi W, Smyth GK (2015) limma powers differential expression analyses for RNA-sequencing and microarray studies. Nucleic Acids Res 43:e47.

21. Nestler EJ (2015) $\Delta$ FosB: a transcriptional regulator of stress and antidepressant responses. Eur J Pharmacol 753:66-72.

22. Renthal W, Kumar A, Xiao G, Wilkinson M, Covington HE 3rd, Maze I, Sikder D, Robison AJ, LaPlant Q, Dietz DM, Rus- so SJ, Vialou V, Chakravarty S, Kodadek TJ, Stack A, Kabbaj M, Nestler EJ (2009) Genome-wide analysis of chromatin regulation by cocaine reveals a role for sirtuins. Neuron 62:335348.

23. Duclot F, Kabbaj M (2017) The role of early growth response 1 (EGR1) in brain plasticity and neuropsychiatric disorders. Front Behav Neurosci 11:35.

24. Wang G, Li S, Gilbert J, Gritton HJ, Wang Z, Li Z, Han X, Selkoe DJ, Man HY (2017) Crucial roles for SIRT2 and AMPA receptor acetylation in synaptic plasticity and memory. Cell Reports 20:1335-1347.

25. Kazantsev AG, Thompson LM (2008) Therapeutic application of histone deacetylase inhibitors for central nervous system disorders. Nat Rev Drug Discov 7:854-868.

26. Cadet JL, Jayanthi S (2013) Epigenetics of methamphetamine-induced changes in glutamate function. Neuropsychopharmacology 38:248-249.

27. McEwen BS, Nasca C, Gray JD (2016) Stress effects on neuronal structure: hippocampus, amygdala, and prefrontal cortex. Neuropsychopharmacology 41:3-23.

28. Thiel G, Schoch S, Petersohn D (1994) Regulation of synapsin I gene expression by the zinc finger transcription factor zif268/egr-1. J Biol Chem 269:15294-15301.

29. Shi YJ, Matson C, Lan F, Iwase S, Baba T, Shi Y (2005) Regulation of LSD1 histone demethylase activity by its associated factors. Mol Cell 19:857-864.

30. Wang DY, Kosowan J, Samsom J, Leung L, Zhang KL, Li YX, Xiong Y, Jin J, Petronis A, Oh G, Wong AH (2018) Inhibition of the G9a/GLP histone methyltransferase complex modulates anxiety-related behavior in mice. Acta Pharmacol Sin 39:866-874.

31. Kallarackal AJ, Kvarta MD, Cammarata E, Jaberi L, Cai X, Bailey AM, Thompson SM (2013) Chronic stress induces a selective decrease in AMPA receptor-mediated synaptic excitation at hippocampal temporoammonic-CA1 synapses. J Neurosci 33:15669-15674. 